\title{
Individual and Combined Effect of Diabetes and Lifetime Criminal Justice Involvement on Healthcare Utilization in US Adults, 2015-2019
}

\author{
Laura C. Hawks, MD, MPH',2, Rebekah J. Walker, $P h D^{1,2}$, and Leonard E. Egede, MD MS \\ ${ }^{1}$ Division of General Internal Medicine, Medical College of Wisconsin, 8701 Watertown Plank Rd., Milwaukee,WI 53226-3596, USA; ${ }^{2}$ Center for Advancing Popula- \\ tion Science, Medical College of Wisconsin, Milwaukee,WI, USA. .
}

Background: Diabetes and criminal justice involvement (CJI) are both associated with poor health outcomes and increased healthcare utilization. However, little is known about the additive effects of these risk factors when combined. This study examined the individual and combined effects of diabetes and CJI on healthcare utilization.

Methods: Data from the National Survey of Drug Use and Health (2015-2019) was used to create a crosssectional, nationally representative sample of US adults with diabetes, CJI, combination of both, or neither. Negative binomial regression was used to test the association between those with CJI and diabetes (compared to diabetes alone) and three utilization types (outpatient, ED, and inpatient) controlling for relevant sociodemographic and clinical covariates.

Results: Of 212,079 respondents, representing $268,893,642$ US adults, $8.8 \%$ report having diabetes alone, $15.2 \%$ report having CJI alone, and $1.8 \%$ report both diabetes and lifetime CJI. After adjustment, those with diabetes and CJI had increased acute care utilization compared to those with diabetes alone (ED visits: IRR 1.13; 95\% CI 1.00-1.28; nights hospitalized: IRR 1.34; 95\% CI 1.08-1.67). There was no difference in outpatient utilization between those with both diabetes and CJI compared to those with diabetes alone (IRR 1.04, 95\% CI 0.99-1.10).

Conclusion: Individuals with complex social and health risks such as diabetes and lifetime CJI experience increased acute healthcare utilization but no difference in outpatient utilization. Tailored interventions that target both diabetes and CJI are needed to reduce unnecessary utilization in this population.

KEY WORDS: diabetes - incarceration - criminal justice involvement . healthcare utilization $\cdot$ health services research

J Gen Intern Med 37(7):1688-96

DOI: $10.1007 / \mathrm{s} 11606-021-07218-1$

(C) Society of General Internal Medicine 2021

Received February 17, 2021

Accepted October 15, 2021

Published online February 8, 2022

\section{INTRODUCTION}

In the USA, diabetes mellitus (DM) is a significant contributor to morbidity and mortality, as well as a leading contributor to healthcare utilization and cost. ${ }^{1}$ There are stark racial disparities in diabetes prevalence and outcomes, with Black Americans experiencing prevalence rates $40 \%$ greater than White Americans as well as increased risk of complications and death. ${ }^{2}$ The COVID-19 pandemic has laid bare the preexisting health inequities imposed by structural racism, resulting in increased attention and burgeoning commitment to study the ways social risks layer onto health risks such that minority groups experience worse health outcomes for conditions such as diabetes. ${ }^{3,4}$

Criminal justice involvement (CJI) is a significant social risk factor for morbidity and mortality ${ }^{5,6}$, and one experienced disproportionately by Black people in the USA. While Black American men constitute only $13 \%$ of the adult male population, they make up $40 \%$ of those incarcerated. ${ }^{7}$ Those with CJI experience higher rates of infectious as well as noncommunicable disease, including an estimated $40 \%$ increased risk of diabetes. ${ }^{8}$ Those returning to the community from prison or jail, and those under community supervision, experience higher mortality rates than their age-matched peers. ${ }^{5,9}$ Even limited exposure to the criminal justice system, such as being stopped by the police, is associated with worse overall well-being and deterioration in physical and mental health. ${ }^{10}$ Examining the relationship between these overlapping risks faced disproportionately by minorities in the USA will be necessary to reduce disparities in health outcomes.

Prior work has examined the association between diabetes and healthcare utilization and CJI and healthcare utilization. ${ }^{11,12}$ Adults with diabetes, known to have higher rates of outpatient and acute care utilization, must engage with outpatient management to achieve diabetes control, thereby reducing risk of complications and overall healthcare costs. ${ }^{13,14}$ Those with CJI experience an increased likelihood of ED visits and hospitalization but decreased rates of outpatient utilization. ${ }^{15-17}$ Increased acute care utilization may indicate poor disease control, especially among those with chronic illness. One way that CJI undermines health, especially in those with chronic disease, is by limiting access to employment (and thereby employer-sponsored insurance), which may decrease access to primary care. ${ }^{18}$ Indeed, one study established an 
association between lower outpatient healthcare utilization and CJI but was not stratified by type of chronic disease. ${ }^{19}$ It is possible that decreased access to preventive outpatient care may contribute to increased acute care utilization among those with CJI, particularly those with underlying diabetes.

However, little is known about the combined effects of CJI and diabetes on healthcare utilization. To better understand the individual and combined effects of diabetes and criminal justice involvement on outpatient or acute care utilization, this study used data from the National Survey of Drug Use and Health (NSDUH) to study the association between diabetes, lifetime CJI, neither, or both on utilization outcomes. We hypothesized that compared with diabetes alone, the combination of diabetes and criminal justice involvement would be associated with an additive increased rate of acute care utilization.

\section{METHODS}

We used data from the NSDUH (2015-2019), the Substance Abuse and Mental Health Services Administration's (SAMHSA) annual, nationally representative, cross-sectional survey which provides annual epidemiologic data on the prevalence of substance use and mental health conditions and gathers substantial amounts of associated information, including some measures of chronic health and criminal justice involvement. While interviews are conducted in person, the majority of responses are provided directly into a computer. During the included study years, the response rate was $66-69 \% .{ }^{20}$ The survey is administered to participants aged 12 and older. We included in our sample all respondents aged 18 years and older.

\section{Main Measures}

To study the individual and combined effects of diabetes and lifetime CJI, we created four mutually exclusive categories to describe diabetes and lifetime CJI status:

1. Respondents with neither diabetes nor CJI,

2. Respondents with CJI and no diabetes (hereafter referred to as CJI alone),

3. Respondents with diabetes alone and no CJI (hereafter referred to as diabetes alone),

4. Respondents with both diabetes and CJI.

We identified respondents with lifetime CJI as those who responded "yes" to the question, "Not counting minor traffic violations, have you ever been arrested and booked for breaking the law? Being 'booked' means that you were taken into custody and processed by the police or by someone connected with the courts, even if you were then released." We identified respondents as having diabetes if they responded "yes" to the question, "Have you ever been told by a doctor or other healthcare professional that you have diabetes or sugar diabetes?"

\section{Outcomes}

Our dependent variables included three quantitative measures of outpatient, ED, and inpatient healthcare utilization during the prior 12 months. To assess the number of outpatient visits, we examined responses to the question: "During the past 12 months, how many times have you visited a doctor, nurse, physician assistant, or nurse practitioner about your own health at a doctor's office, a clinic, or some other place?" To assess the number of emergency department visits, we examined responses to the question: "During the past 12 months, how many different times have you been treated in an emergency room for any reason?" Finally, to measure nights of inpatient hospitalization, we examined responses to the question: "During the past 12 months, how many nights were you an inpatient in a hospital?"

\section{Covariates}

A priori, we considered additional covariates based on identification of potential sociodemographic and clinical confounders. The following demographic covariates were used: age (categorized into 18-25, 26-34, 35-49, and 50+ years); self-reported race/ethnicity (non-Hispanic white, non-Hispanic Black, Hispanic, other race/ethnicity [nonHispanic Native American/Alaskan Native, non-Hispanic Native Hawaiian or Pacific Islander; non-Hispanic Asian; or more than one race]); self-reported gender (male, female); poverty level $(<100 \%$ federal poverty level as determined by the Census Bureau, 100-200\% poverty level, $>200 \%$ poverty level); educational status (less than high school, high school degree or equivalent, some college, or college graduate); marital status (married, widowed/divorced/separated, never married); current employment status (full time, part time, unemployed, other [disabled, keeping house full-time; in school/training; retired; some other reason]); and insurance coverage (Medicare, Medicaid, VA healthcare, private, none).

Clinical covariates included a number of physical and mental health comorbidities. Physical comorbidities were identified by self-report, and included high blood pressure, heart condition, kidney disease, asthma, COPD, cirrhosis, hepatitis B/C, HIV/AIDS, and cancer. Mental health comorbidities included substance use disorder and mental illness. Both past year substance use disorder (SUD) and any mental health illness (AMI) were created using a validated series of questions within the dataset, which diagnostically predicts SUD or AMI. ${ }^{21}$ Using DSM-IV criteria, the NSDUH model generates a diagnostic variable for substance abuse or dependence (now considered substance use disorder) including use of the following substances: alcohol, marijuana, cocaine, heroin, hallucinogens, inhalants, methamphetamine, pain relievers, tranquilizers, stimulants, or sedatives. ${ }^{22}$ A similar NSDUH model uses a validated series of mental health questions to diagnostically predict presence 
of any mental illness. ${ }^{23}$ Both were treated as a dichotomous variable in the analysis.

\section{Statistical Analyses}

We first used Pearson's chi-square statistics to compare the sociodemographic characteristics and health profiles of our four groups of respondents. Secondly, we calculated the crude mean number (and 95\% CI) of outpatient visits, ED visits, and nights hospitalized for each of the four groups. We then calculated unadjusted incident rate ratios (IRRs) using univariate negative binomial regression models for which the 4-category variable for diabetes and lifetime CJI status was the independent variable (those with diabetes alone serving as the reference group) and frequency of healthcare utilization was the dependent variable. Each utilization outcome (number of outpatient visits, ED visits, and nights hospitalized) was run as a separate model. Finally, multivariate negative binomial regression was used to calculate IRRs for the relationship between the 4-category diabetes and lifetime CJI status variable (those with diabetes alone again serving as the reference group) and three separate outcomes (number of outpatient visits, ED visits, and nights hospitalized). Models were adjusted for age, sex, race, poverty level, education, marital status, employment, health insurance coverage type, and comorbid medical, substance use, and mental health conditions.

We conducted all analyses with Stata version 16 (Stata Corp LP, College Station, TX) and used weights provided by SAMHSA that account for complex survey design and allow extrapolation for the US population as a whole. Survey weights were adjusted to account for combining multiple years of survey data in accordance with SAMHSA instructions (the final weight divided by the number of years of combined data; in this case, 5). We considered 2 -sided $p$-values of less than 0.05 to be statistically significant.

\section{RESULTS}

The unweighted sample included 212,893 respondents, representing 268,983,642 US adults who participated in the most recent years (2015-2019) of the NSDUH. In this nationally representative sample, over a quarter of respondents reported at least one exposure: $8.8 \%$ diabetes alone, $15.2 \%$ CJI alone, and 1.8\% diabetes and lifetime CJI. Demographic characteristics by diabetes and CJI status are displayed in Table 1. Black respondents disproportionately reported having both diabetes and prior CJI compared to neither risk factor $(18.1 \% \%$ versus $10.7 \% p<0.001)$ as did those with income $<100 \%$ FPL $(19.7 \%$ versus $12.7 \%, p<0.001)$, those with less than a high school education $(20.1 \%$ versus $10.9 \%, p<0.001)$, and those with Medicaid $(27.1 \%$ versus $12.3 \%, p<0.001$ ), Medicare (39.3\% versus $20.5 \%, p<0.001$ ), or VA Health $(10.9 \%$ versus $4.5 \% p<0.001)$. Those with both diabetes and lifetime CJI were most likely to have multiple measured comorbid medical conditions, with the highest prevalence of comorbid disease being hypertension (42.9\%), past-year any mental illness (31.6\%), or a heart condition (26.4\%).

Table 2 reports the unadjusted mean number of outpatient visits, ED visits, or nights hospitalized in the past 12 months. For outpatient utilization, those with neither CJI or CJI only reported similar number of outpatient visits (mean 3.1 [95\% CI 3.1-3.2] and 3.0 [95\% CI 2.9-3.1], respectively); and there was no difference between the mean number of visits for those with diabetes alone or diabetes plus CJI (5.6 [95\% CI 5.5-57] and 5.7 [95\% CI 5.4-5.9], respectively. However, there were differences between all four groups for both measures of acute care utilization. For ED visits, those with neither diabetes nor CJI reported the lowest number of ED visits $(0.43,95 \%$ CI $0.42-0.44)$, with CJI alone reporting the second fewest $(0.62,95 \%$ CI $0.59-0.64)$, diabetes alone reporting more $(0.75,95 \%$ CI $0.71-0.79)$, and those with both diabetes and CJI reporting the most (1.01, 95\% CI 0.91-1.10). The same pattern persisted for nights hospitalized. Those with neither diabetes nor CJI reported the fewest $(0.34,95 \%$ CI $0.32-0.35)$, those with CJI reporting the second fewest $(0.54,95 \%$ CI $0.50-0.59)$, those with diabetes alone report more (1.11, 95\% CI, 1.01-1.20), and those with both diabetes and CJI reporting the most $(1.44,95 \%$ CI 1.24-1.65).

Table 3 reports the results of unadjusted and adjusted negative binomial regression models for healthcare utilization, in which diabetes alone served as the reference group. In models both unadjusted and fully adjusted for sociodemographic covariates and comorbid medical conditions, having both diabetes and prior CJI was associated with increased ED visits and night hospitalized, but not increased outpatient visits when compared to those having diabetes alone. Compared to those with diabetes alone, those with both diabetes and CJI experienced an IRR of 1.04 (95\% CI 0.99-1.10) for outpatient utilization, an IRR of 1.13 (95\% CI 1.00-1.28; $p=0.04$ ) for ED visits, and an IRR of 1.34 (95\% CI 1.08-1.67) for inpatient nights. Those with neither diabetes nor CJI or CJI alone experienced a lower IRR for all three utilization categories when compared to those with diabetes alone. In these fully adjusted models, Black race was consistently strongly associated with higher rates of acute care utilization, as was lower socioeconomic status. The comorbid medical conditions most strongly associated with increased ED or inpatient utilization were heart conditions (IRR 1.56, 95\% CI: 1.47-1.66; IRR 2.42, 95\% CI 2.14, 2.74, respectively); kidney disease (IRR $1.53,95 \%$ CI: 1.38 , 1.69; IRR 1.96, 95\% CI: 1.72, 2.22, respectively); and pastyear AMI (IRR 1.52, 95\% CI: 1.44, 1.61; IRR 1.84, 95\% CI $1.65,2.06$, respectively). 
Table 1 Demographics of US Adults by Diabetes Status and Lifetime Criminal Justice Involvement (CJI), 2015-2019*

\begin{tabular}{|c|c|c|c|c|}
\hline & $\begin{array}{l}\text { Neither diabetes } \\
\text { nor CJI } \\
(N=163,003)\end{array}$ & $\begin{array}{l}\text { CJI alone } \\
(N=34,537)\end{array}$ & $\begin{array}{l}\text { Diabetes alone } \\
(N=11,797)\end{array}$ & $\begin{array}{l}\text { Both dia- } \\
\text { betes and } \\
\text { CJI } \\
(N=2742)\end{array}$ \\
\hline & $\%$ & $\%$ & $\%$ & $\%$ \\
\hline \multicolumn{5}{|l|}{ Age } \\
\hline $18-25$ & 16.2 & 10.5 & 2.0 & 1.7 \\
\hline $26-34$ & 16.5 & 21.4 & 3.9 & 5.2 \\
\hline $35-49$ & 24.3 & 31.5 & 15.5 & 23.3 \\
\hline $50+$ & 42.8 & 36.5 & 78.4 & 79.6 \\
\hline \multicolumn{5}{|l|}{ Sex } \\
\hline Male & 43.6 & 70.7 & 43.2 & 72.3 \\
\hline Female & 56.4 & 29.2 & 56.7 & 27.6 \\
\hline \multicolumn{5}{|l|}{ Race } \\
\hline White NH & 64.3 & 65.9 & 60.0 & 60.0 \\
\hline Black NH & 10.7 & 14.8 & 14.7 & 18.1 \\
\hline Hispanic & 16.2 & 13.9 & 17.2 & 14.7 \\
\hline Other & 8.7 & 5.2 & 8.0 & 7.1 \\
\hline \multicolumn{5}{|l|}{ Poverty level } \\
\hline$<100 \%$ & 12.7 & 18.8 & 13.9 & 19.7 \\
\hline $100-200 \%$ & 18.4 & 22.4 & 24.3 & 6.5 \\
\hline$>200 \%$ & 68.7 & 58.6 & 61.7 & 53.6 \\
\hline \multicolumn{5}{|l|}{ Education } \\
\hline$<$ high school & 10.9 & 16.0 & 18.3 & 20.1 \\
\hline HS grad & 23.1 & 29.8 & 27.8 & 32.4 \\
\hline Some college & 30.2 & 34.7 & 29.6 & 32.3 \\
\hline College grad & 35.6 & 19.4 & 24.1 & 15.1 \\
\hline \multicolumn{5}{|l|}{ Marital status } \\
\hline Married & 53.0 & 40.2 & 59.3 & 48.8 \\
\hline Divorced/widowed & 17.2 & 24.2 & 29.0 & 32.1 \\
\hline Never married & 29.6 & 35.7 & 11.6 & 19.6 \\
\hline \multicolumn{5}{|l|}{ Employment } \\
\hline Full time & 50.6 & 56.7 & 31.4 & 34.1 \\
\hline Part time & 14.1 & 10.4 & 10.3 & 8.6 \\
\hline Unemployed & 3.9 & 6.9 & 2.7 & 4.4 \\
\hline Other** & 31.3 & 25.9 & 55.5 & 52.7 \\
\hline \multicolumn{5}{|l|}{ Health insurance } \\
\hline Private & 70.1 & 54.5 & 63.1 & 49.1 \\
\hline Medicaid & 12.3 & 21.1 & 17.7 & 27.1 \\
\hline Medicare & 20.5 & 14.3 & 49.1 & 39.3 \\
\hline VA Health & 4.5 & 5.5 & 8.8 & 10.9 \\
\hline Uninsured & 9.2 & 16.2 & 4.6 & 7.7 \\
\hline \multicolumn{5}{|l|}{ Comorbidities } \\
\hline Heart condition & 8.8 & 9.1 & 23.6 & 26.4 \\
\hline Hypertension & 16.7 & 15.7 & 45.9 & 42.9 \\
\hline Kidney disease & 1.4 & 1.2 & 6.8 & 6.6 \\
\hline COPD & 3.2 & 5.7 & 9.7 & 12.4 \\
\hline Asthma & 9.2 & 9.5 & 11.0 & 11.4 \\
\hline Cirrhosis & 0.1 & 0.5 & 1.0 & 1.6 \\
\hline Hepatitis B/C & 0.8 & 3.1 & 1.5 & 4.3 \\
\hline HIV/AIDS & 0.1 & 0.5 & 0.2 & 0.4 \\
\hline Cancer & 6.0 & 4.4 & 11.1 & 9.8 \\
\hline Past-year SUD*** & 5.9 & 19.9 & 2.7 & 11.9 \\
\hline Past-year AMI**** & 17.4 & 25.8 & 19.2 & 31.6 \\
\hline
\end{tabular}


Table 1 (continued)

Table 2 Crude Mean Number of Outpatient Visits, ED Visits, or Nights Hospitalized in Past 12 Months Among US Adults with Diabetes, Lifetime Criminal Justice Involvement, Both, or Neither, 2015-2019
*Significant differences at $p<0.0001$ existed for all variables
** Other employment status includes disabled, keeping house full-time; in school/training; retired; some other reason
${ }^{* * *}$ SUD substance use disorder including alcohol, marijuana, cocaine, heroin, hallucinogens, inhalants, meth- amphetamine, pain relievers, tranquilizers, stimulants, or sedatives
****A AMI any mental illness as defined by NSDUH predictive model

\begin{tabular}{lccc}
\hline & $\begin{array}{l}\text { Outpatient visits } \\
\text { Mean }(95 \% \mathrm{CI})\end{array}$ & $\begin{array}{l}\text { ED visits } \\
\text { Mean }(95 \% \mathrm{CI})\end{array}$ & $\begin{array}{l}\text { Inpatient nights } \\
\text { Mean (95\% CI) }\end{array}$ \\
\hline Neither diabetes nor CJI & $3.1(3.1-3.2)$ & $0.43(0.42-0.44)$ & $0.34(0.32-0.35)$ \\
CJI alone & $3.0(2.9-3.1)$ & $0.62(0.59-0.64)$ & $0.54(0.50-0.59)$ \\
Diabetes alone & $5.6(5.5-5.7)$ & $0.75(0.71-0.79)$ & $1.11(1.01-1.20)$ \\
Both diabetes and CJI & $5.7(5.4-5.9)$ & $1.01(0.91-1.10)$ & $1.44(1.24-1.65)$ \\
\hline
\end{tabular}

\section{DISCUSSION}

In this nationally representative sample, both lifetime criminal justice involvement and diabetes were common, with a quarter of respondents reporting at least one exposure. Having both exposures was associated with higher rates of ED and inpatient visits relative to having diabetes alone in both unadjusted and fully adjusted models. However, there was no difference in outpatient utilization between these two groups. Similarly, those with CJI but not diabetes had higher rates of acute care utilization and no difference in outpatient utilization compared to those with neither exposure. Consistent with our hypothesis of overlapping social risks in this population, Black men and those of low socioeconomic status were overrepresented among those with both lifetime $\mathrm{CJI}$ and diabetes. Additionally, those with both diabetes and lifetime CJI reported high rates of comorbid heart disease, hypertension, COPD, substance use, and past-year mental illness. However, the additional risk of acute care utilization due to lifetime CJI among those with diabetes persisted in models adjusted for sociodemographic covariates and comorbid medical conditions.

The medical literature has rarely attempted to evaluate the individual and cumulative effects that individuals exposed to multiple layers of social and health risks experience. To our knowledge, no prior studies have analyzed the additive effect of CJI and chronic disease, and this is the first study analyzing the individual or combined effects of diabetes and CJI on healthcare utilization. While it is well known that individuals with diabetes have higher rates of outpatient, ED, and inpatient utilization, and those with CJI have higher rates of ED and inpatient utilization ${ }^{14,15,24}$, our findings contextualize how CJI and diabetes function as combined risk factors for increased utilization of healthcare, especially acute care. The results show that individuals with either diabetes or CJI as a single risk factor are at higher risk of acute care utilization compared to those with neither. Moreover, there is an additive effect on acute care utilization when both risk factors occur simultaneously.
Our multivariate regression analyses highlighted other factors correlated with increased acute care utilization for those with both diabetes and CJI. In our fully adjusted model, comorbid heart disease, comorbid kidney disease, mental illness, and substance use disorder were independently associated with increased ED visits and hospitalization. Those with CJI have increased rates of cardiovascular disease, mental illness, and substance use disorder. ${ }^{6,25,26}$ Studies suggest that both CJI and diabetes have a bidirectional causal relationship with mental illness. Mental illness increases risk for and is exacerbated by both CJI and diabetes and is associated with worse diabetes outcomes. ${ }^{27,28}$ Similarly, untreated substance use disorder has been shown to undermine glycemic control and is associated with increased likelihood of stroke, diabetic neuropathy, renal disease, and mortality in patients with diabetes. ${ }^{29}$ Therefore, targeted interventions to treat these comorbidities may be crucial to achieving glycemic control and preventing unnecessary acute care utilization in patients with social risk factors.

Contrary to our hypothesis that those with CJI would experience decreased outpatient utilization, we found that those with CJI and diabetes had no difference in outpatient utilization compared to those with diabetes alone in unadjusted analyses. In the adjusted analyses, there was a small increase in utilization of marginal significance (IRR 1.04, 95\% CI 0.99-1.10) for those with both exposures compared to those with diabetes alone. Taken in sum, the findings suggest that for those with diabetes, barriers to outpatient care alone cannot explain the relationship between CJI and increased acute care utilization. It is possible that those with CJI experience different quality of care due to structural barriers or access to different health systems ${ }^{19}$, although we are unable to further explore this question using NSDUH data.

Race/ethnicity is also strongly independently associated with increased acute care utilization-even after controlling for other indicators of socioeconomic status. These findings suggest the role of structural racism, a potentially modifiable risk factor, in the disparities identified in this 
Table 3 Unadjusted and Adjusted Multivariate Negative Binomial Regression for Relationship Between Emergency Department Visits, Inpatient Stays, and Outpatient Utilization Among Adults with Diabetes, Lifetime Exposure to the Criminal Justice System, or Neither Both [Incident Risk Ratio with 95\% Confidence Interval]

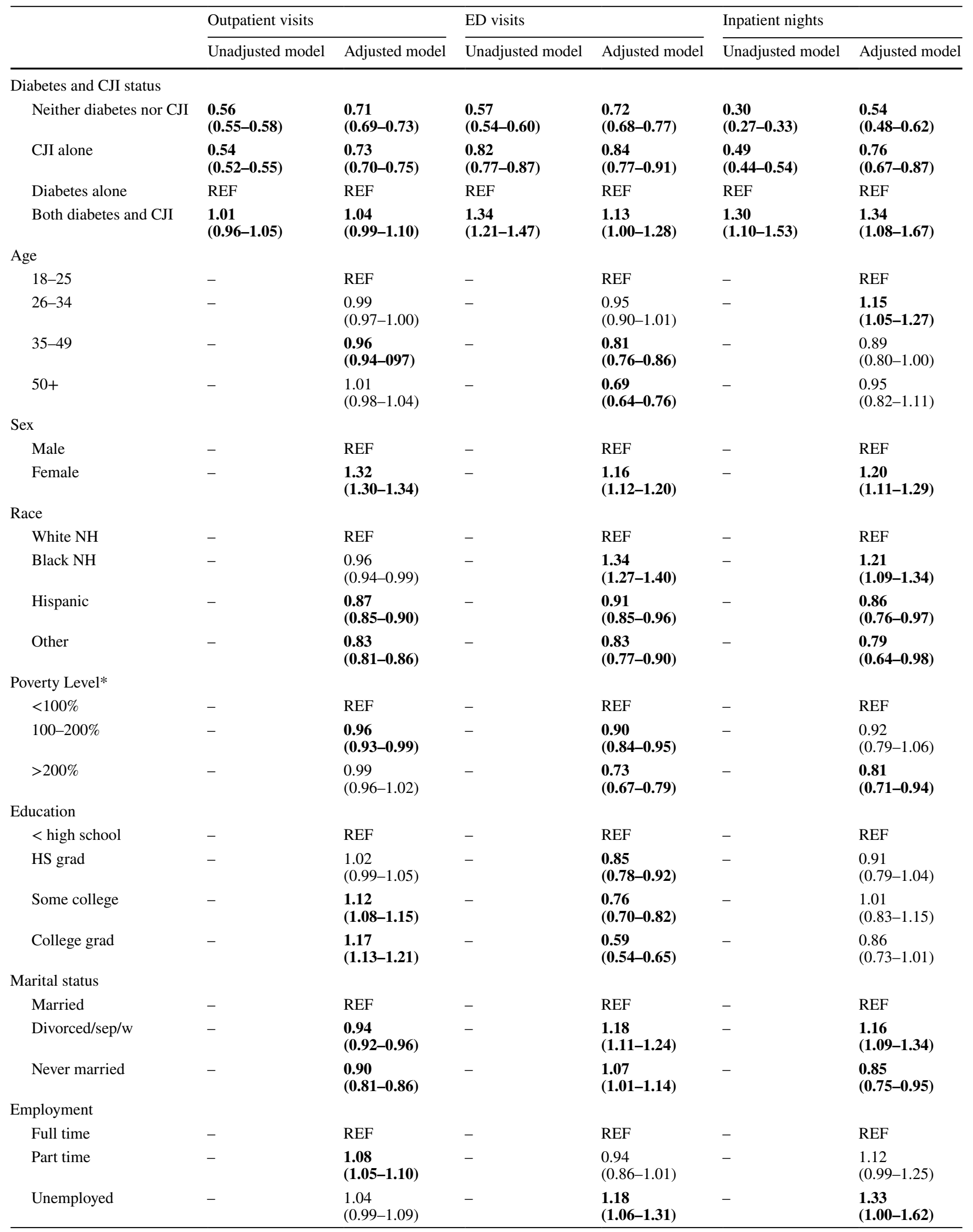


Table 3 (continued)

\begin{tabular}{|c|c|c|c|c|c|c|}
\hline & Outpatient visits & & ED visits & & Inpatient nights & \\
\hline & Unadjusted model & Adjusted model & Unadjusted model & Adjusted model & Unadjusted model & Adjusted model \\
\hline Other* & - & $\begin{array}{l}1.20 \\
(1.17-1.23)\end{array}$ & - & $\begin{array}{l}1.13 \\
(1.07-1.20)\end{array}$ & - & $\begin{array}{l}1.88 \\
(1.65-2.13)\end{array}$ \\
\hline Health insurance & & & & & & \\
\hline Private & - & $\begin{array}{l}0.99 \\
(0.95-1.02)\end{array}$ & - & $\begin{array}{l}0.89 \\
(0.83-0.95)\end{array}$ & - & $\begin{array}{l}0.93 \\
(0.80-1.09)\end{array}$ \\
\hline Medicaid & - & $\begin{array}{l}1.19 \\
(1.14-1.23)\end{array}$ & - & $\begin{array}{l}1.41 \\
(1.32-1.51)\end{array}$ & - & $\begin{array}{l}1.65 \\
(1.41-1.93)\end{array}$ \\
\hline Medicare & - & $\begin{array}{l}1.10 \\
(1.06-1.13)\end{array}$ & - & $\begin{array}{l}1.22 \\
(1.14-1.31)\end{array}$ & - & $\begin{array}{l}1.49 \\
(1.28-1.73)\end{array}$ \\
\hline VA Health & - & $\begin{array}{l}1.14 \\
(1.10-1.19)\end{array}$ & - & $\begin{array}{l}1.27 \\
(1.11-1.47)\end{array}$ & - & $\begin{array}{l}1.34 \\
(1.11-1.62)\end{array}$ \\
\hline Uninsured & - & $\begin{array}{l}0.64 \\
(0.61-0.67)\end{array}$ & - & $\begin{array}{l}0.93 \\
(0.86-1.01)\end{array}$ & - & $\begin{array}{l}0.79 \\
(0.64-0.98)\end{array}$ \\
\hline Comorbidities & & & & & & \\
\hline Heart condition & - & $\begin{array}{l}1.39 \\
(1.36-1.42)\end{array}$ & - & $\begin{array}{l}1.56 \\
(1.47-1.66)\end{array}$ & - & $\begin{array}{l}2.42 \\
(2.14-2.74)\end{array}$ \\
\hline Hypertension & - & $\begin{array}{l}1.25 \\
(1.22-1.28)\end{array}$ & - & $\begin{array}{l}1.07 \\
(1.01-1.12)\end{array}$ & - & $\begin{array}{l}1.20 \\
(1.07-1.36)\end{array}$ \\
\hline Kidney disease & - & $\begin{array}{l}1.32 \\
(1.25-1.39)\end{array}$ & - & $\begin{array}{l}1.53 \\
(1.38-1.69)\end{array}$ & - & $\begin{array}{l}1.96 \\
(1.72-2.22)\end{array}$ \\
\hline COPD & - & $\begin{array}{l}1.25 \\
(1.21-1.29)\end{array}$ & - & $\begin{array}{l}1.40 \\
(1.29-1.52)\end{array}$ & - & $\begin{array}{l}1.70 \\
(1.51-1.90)\end{array}$ \\
\hline Asthma & - & $\begin{array}{l}1.22 \\
(1.19-1.25)\end{array}$ & - & $\begin{array}{l}1.26 \\
(1.20-1.32)\end{array}$ & - & $\begin{array}{l}1.05 \\
(0.93-1.17)\end{array}$ \\
\hline Cirrhosis & - & $\begin{array}{l}1.27 \\
(1.12-1.44)\end{array}$ & - & $\begin{array}{l}1.55 \\
(1.08-2.22)\end{array}$ & - & $\begin{array}{l}2.12 \\
(1.46-3.08)\end{array}$ \\
\hline Hepatitis B/C & - & $\begin{array}{l}1.18 \\
(1.09-1.28)\end{array}$ & - & $\begin{array}{l}1.11 \\
(0.96-1.30)\end{array}$ & - & $\begin{array}{l}1.48 \\
(0.98-2.22)\end{array}$ \\
\hline HIV/AIDS & - & $\begin{array}{l}1.54 \\
(1.35-1.76)\end{array}$ & - & $\begin{array}{l}1.22 \\
(0.93-1.60)\end{array}$ & - & $\begin{array}{l}2.07 \\
(0.82-5.23)\end{array}$ \\
\hline Cancer & - & $\begin{array}{l}1.44 \\
(1.39-1.49)\end{array}$ & - & $\begin{array}{l}1.12 \\
(1.04-1.19)\end{array}$ & - & $\begin{array}{l}2.14 \\
(1.84-2.49)\end{array}$ \\
\hline Past-year SUD** & - & $\begin{array}{l}1.04 \\
(1.01-1.07)\end{array}$ & - & $\begin{array}{l}1.23 \\
(1.17-1.29)\end{array}$ & - & $\begin{array}{l}1.18 \\
(1.04-1.33)\end{array}$ \\
\hline Past-year AMI*** & - & $\begin{array}{l}1.43 \\
(1.40-1.46)\end{array}$ & - & $\begin{array}{l}1.52 \\
(1.44-1.61)\end{array}$ & - & $\begin{array}{l}1.84 \\
(1.65-2.06)\end{array}$ \\
\hline
\end{tabular}

Models adjusted for age, sex, race, poverty level, education, marital status, employment, health insurance coverage type, and comorbid medical, substance use, and mental health conditions

* Other employment status includes disabled, keeping house full-time; in school/training; retired; some other reason

${ }^{* *}$ SUD substance use disorder including alcohol, marijuana, cocaine, heroin, hallucinogens, inhalants, methamphetamine, pain relievers, tranquilizers, stimulants, or sedatives

*** AMI any mental illness as defined by NSDUH predictive model

Items in bold signify p-value $<0.05$

study. ${ }^{30,31}$ Prior work has identified measures of socioeconomic status-both low income and low educationas indicators of increased acute care utilization among patients with diabetes ${ }^{32,33}$, but structural racism likely plays a role in how socioeconomic status influences racial/ ethnic disparities as well. For example, perceived intrapersonal discrimination has been generally linked with poor health outcomes in Black patients, which may influence acute care utilization. ${ }^{34}$ Moreover, structural forces such as residential segregation, which leads to unique barriers to healthy food and transportation, may also contribute to increased acute care utilization, especially for patients with diabetes. ${ }^{35}$ Identifying the numerous ways that structural racism impacts the healthcare outcomes of minorities in the USA is an important first step to reducing healthcare disparities. Based on these findings, developing interventions that target the combined social and health risks experienced by racial/ethnic minorities may be one way in which to move the field forward toward solutions. 
Our paper has several limitations worth noting. First, as a cross-sectional sample, our analyses cannot establish casual association. Secondly, because our data comes from a national survey, we are unable to distinguish different types or qualities of outpatient care. It is possible that those with CJI involvement receive more outpatient care that does not focus on diabetes management (such a primary care-based treatment for substance use disorder). Thirdly, criminal justice involvement and chronic medical conditions are self-reported, along with all other covariates. Prior research shows self-reported justice involvement tracks closely or better than administrative data ${ }^{36}$; however, health conditions may be under-reported especially by those with less contact with the healthcare system. Finally, while healthcare utilization is a useful outcome in its own right, our research cannot comment on health outcomes due to the design of the survey. Future research should evaluate the role of healthcare utilization in clinical outcomes including glycosylated hemoglobin levels and rates of complications among those with diabetes and CJI compared to diabetes alone.

In conclusion, our study found that individuals with CJI and diabetes face compound layers of social and health risk, and those with both factors experience particularly high rates of acute care utilization. By identifying modifiable risk factors for those with CJI and diabetes, we can study meaningful interventions to reduce unnecessary healthcare utilization and increase regular engagement with the healthcare system.

Corresponding Author Leonard E. Egede, MD, MS; 1Division of General Internal Medicine, Medical College of Wisconsin, 8701 Watertown Plank Rd., Milwaukee, WI 53226-3596, USA (e-mail: legede@mcw.edu)

Author Contribution LH, RJW, and LEE designed the study. LH acquired the data and LEE analyzed the data. LH drafted the manuscript. RJW and LEE reviewed and revised the manuscript. All authors critically revised for intellectual content and approved the final manuscript. LH and LEE are guarantors of the work.

Funding Effort for this study was partially supported by the National Institute of Diabetes and Digestive Kidney Disease (K24DK093699, R01DK118038, R01DK120861, PI: Egede) and the National Institute for Minority Health and Health Disparities (R01MD013826, PI: Egede/ Walker).

Data Availability The dataset supporting the conclusions of this article are publicly available from the National Survey of Drug Use and Health website: https://www.datafiles.samhsa.gov/study-series/national-survey-drug-use-and-health-nsduh-nid13517.

The Medical College of Wisconsin's Institutional Review Board deemed this study exempt from review because the data are deidentified and publicly available.

Consent for Publication Not applicable.
Conflict of Interest The authors declare that they do not have a conflict of interest.

\section{REFERENCES}

1. Centers for Disease Control Prevention. National Diabetes Statistics Report, 2020. Atlanta, GA: Centers for Disease Control and Prevention, US Department of Health and Human Services Web site. https://www.cdc.gov/diabetes/pdfs/data/stati stics/national-diabetes-statistics-report.pdf. Published 2020. Accessed 21 Jan 2021.

2. Clements JM, West BT, Yaker Z, et al. Disparities in diabetes-related multiple chronic conditions and mortality: The influence of race. Diabetes Research and Clinical Practice. 2020;159:107984. doi:https://doi.org/https://doi.org/10. 1016/j.diabres.2019.107984. Accessed 01 Jan 2020.

3. Egede LE, Walker RJ. Structural racism, social risk factors, and Covid-19-a dangerous convergence for black Americans. New England Journal of Medicine. 2020;383(12):e77.

4. Bibbins-Domingo K. This Time Must Be Different: Disparities During the COVID-19 Pandemic. Annals of Internal Medicine. 2020;173(3):233-234. doi:https://doi.org/https://doi.org/10. 7326/M20-2247

5. Binswanger IA, Stern MF, Deyo RA, et al. Release from prison-a high risk of death for former inmates. New England Journal of Medicine. 2007;356(2):157-165.

6. Wilper AP, Woolhandler S, Boyd JW, et al. The health and health care of US prisoners: results of a nationwide survey. Am J Public Health. 2009;99(4):666-672. doi:https://doi.org/ 10.2105/ajph.2008.144279.

7. Sawyer W, Wagner P. Mass incarceration: The whole pie 2020. Prison Policy Initiative Web site. https://www.prisonpolicy.org/ factsheets/pie2020_allimages.pdf. Published 2020. Accessed 21 Jan $2021,24$.

8. Binswanger IA, Krueger PM, Steiner JF. Prevalence of chronic medical conditions among jail and prison inmates in the USA compared with the general population. $J$ Epidemiol Community Health. 2009;63(11):912-919. doi:https://doi.org/10.1136/ jech.2009.090662.

9. Wildeman C, Goldman AW, Wang EA. Age-Standardized Mortality of Persons on Probation, in Jail, or in State Prison and the General Population, 2001-2012. Public Health Reports. 2019;134(6):660-666.

10. Sundaresh R, Yi Y, Roy B, Riley C, Wildeman C, Wang EA. Exposure to the US Criminal Legal System and WellBeing: A 2018 Cross-Sectional Study. Am J Public Health. 2020;110(S1):S1 16-S122. doi:https://doi.org/10.2105/AJPH. 2019.305414..

11. Wang EA, Wang Y, Krumholz HM. A high risk of hospitalization following release from correctional facilities in Medicare beneficiaries: a retrospective matched cohort study, 2002 to 2010. JAMA Intern Med. 2013;173(17):1621-1628. doi:https:// doi.org/10.1001/jamainternmed.2013.9008. Accessed Sep 23.

12. Banerji MA, Dunn JD. Impact of glycemic control on healthcare resource utilization and costs of type 2 diabetes: current and future pharmacologic approaches to improving outcomes. Am Health Drug Benefits. 2013;6(7):382-392.

13. Wolters $\mathbf{R}$, Braspenning $\mathbf{J}$, Wensing $\mathbf{M}$. Impact of primary care on hospital admission rates for diabetes patients: a systematic review. Diabetes research and clinical practice. 2017;129:182-196.

14. Shetty S, Secnik K, Oglesby AK. Relationship of glycemic control to total diabetes-related costs for managed care health plan members with type 2 diabetes. $J$ Manag Care Pharm. 2005; $11(7): 559-564$.

15. Frank JW, Andrews CM, Green TC, Samuels AM, Trinh TT, Friedmann PD. Emergency department utilization among recently released prisoners: a retrospective cohort study. BMC Emerg Med. 2013;13:16. doi:https://doi.org/10.1186/ 1471-227x-13-16.

16. Frank JW, Linder JA, Becker WC, Fiellin DA, Wang EA. Increased hospital and emergency department utilization by 
individuals with recent criminal justice involvement: results of a national survey. $J$ Gen Intern Med. 2014;29(9):1226-1233. doi:https://doi.org/10.1007/s11606-014-2877-y.

17. Young BA, Lin E, Von Korff M, et al. Diabetes complications severity index and risk of mortality, hospitalization, and healthcare utilization. Am J Manag Care. 2008; 14(1):15-23.

18. Nowotny KM, Kuptsevych-Timmer A. Health and justice: framing incarceration as a social determinant of health for Black men in the United States. Sociology Compass. 2018;12(3):e12566.

19. Hawks L, Wang EA, Howell B, et al. Health Status and Health Care Utilization of US Adults Under Probation: 2015-2018. Am J Public Health. 2020;110(9):1411-1417. doi:https://doi.org/10. 2105/ajph.2020.305777.

20. Substance Abuse and Mental Health Services Administration. National Survey on Drug Use and Health: About the survey. https://nsduhweb.rti.org/respweb/about_nsduh.html. Accessed 18 Nov 2020.

21. Substance Abuse and Mental Health Services Administration. 2017 National Survey on Drug Use and Health: Public Use File Codebook. Center for Behavioral Health Statistics and Quality https:// www.datafiles.samhsa.gov/sites/default/files/field-uploads-prote cted/studies/NSDUH-2017/NSDUH-2017-datasets/NSDUH-2017DS0001/NSDUH-2017-DS0001-info/NSDUH-2017-DS0001-infocodebook.pdf. Published 2018. Accessed 31 Jan 2021.

22. Hasin D, Hatzenbuehler ML, Keyes K, Ogburn E. Substance use disorders: diagnostic and statistical manual of mental disorders, (DSM-IV) and International Classification of Diseases, (ICD-10). Addiction. 2006; 101:59-75.

23. Aldworth J, Gulledge K, Warren L, Hedden S, Bose J. National Survey on Drug Use and Health: alternative statistical models to predict mental illness. Rockville, MD: Substance Abuse and Mental Health Services Administration; 2015.

24. American Diabetes Association. Economic consequences of diabetes mellitus in the US in 1997. Diabetes care. 1998;21(2):296-309.

25. Wang EA, Redmond N, Himmelfarb CRD, et al. Cardiovascular Disease in Incarcerated Populations. Journal of the American College of Cardiology. 2017;69(24):2967-2976.

26. Fazel S, Yoon IA, Hayes AJ. Substance use disorders in prisoners: an updated systematic review and meta-regression analysis in recently incarcerated men and women. Addiction. 2017;112(10):1725-1739. doi:https://doi.org/https://doi.org/ 10.1111 /add. 13877 .
27. Sugie NF, Turney $\mathbf{K}$. Beyond incarceration: Criminal justice contact and mental health. American Sociological Review. 2017;82(4):719-743.

28. Rustad JK, Musselman DL, Nemeroff CB. The relationship of depression and diabetes: Pathophysiological and treatment implications. Psychoneuroendocrinology. 2011;36(9): 1276-1286. doi:https://doi.org/https://doi.org/10.1016/j.psyneuen.2011. 03.005 .

29. Winhusen T, Theobald J, Kaelber DC, Lewis D. Medical complications associated with substance use disorders in patients with type 2 diabetes and hypertension: electronic health record findings. Addiction. 2019;114(8):1462-1470. doi:https://doi.org/ 10.1111/add.14607.

30. Hardeman RR, Karbeah JM. Examining racism in health services research: A disciplinary self-critique. Health Serv Res. 2020;55 Suppl 2(Suppl 2):777-780.

31. Boyd RW, Lindo EG, Weeks LD, McLemore MR. On racism: a new standard for publishing on racial health inequities. Health Affairs Blog. 2020;10.

32. Rabi DM, Edwards AL, Southern DA, et al. Association of socioeconomic status with diabetes prevalence and utilization of diabetes care services. BMC health services research. 2006;6(1):124.

33. Booth GL, Hux JE. Relationship Between Avoidable Hospitalizations for Diabetes Mellitus and Income Level. Archives of Internal Medicine. 2003;163(1):101-106.

34. Mays VM, Cochran SD, Barnes NW. Race, race-based discrimination, and health outcomes among African Americans. Annu Rev Psychol. 2007;58:201-225. doi:https://doi.org/10.1146/annur ev.psych.57.102904.190212.

35. Walker RJ, Garacci E, Dawson AZ, Williams JS, Ozieh M, Egede LE. Trends in food insecurity in the United States from 2011-2017: Disparities by age, sex, race/ethnicity, and income. Population health management. 2020.

36. Carroll M, Sutherland G, Kemp-Casey A, Kinner SA. Agreement between self-reported healthcare service use and administrative records in a longitudinal study of adults recently released from prison. Health Justice. 2016;4:11-11. doi:https://doi.org/10. 1186/s40352-016-0042-x.

Publisher's Note Springer Nature remains neutral with regard to jurisdictional claims in published maps and institutional affiliations. 